\title{
Energy Efficiency Maximized Resource Allocation for Opportunistic Relay-Aided OFDMA Downlink with Subcarrier Pairing
}

\author{
Tao Wang $(\mathbb{D}$, Chao Ma, Yanzan Sun, Shunqing Zhang, and Yating Wu \\ Shanghai Institute for Advanced Communication and Data Science, Key laboratory of Specialty Fiber Optics and \\ Optical Access Networks, Joint International Research Laboratory of Specialty Fiber Optics and Advanced Communication, \\ Shanghai University, Shanghai, China \\ Correspondence should be addressed to Tao Wang; twang@shu.edu.cn
}

Received 12 February 2018; Accepted 24 April 2018; Published 29 May 2018

Academic Editor: Paul Honeine

Copyright (C) 2018 Tao Wang et al. This is an open access article distributed under the Creative Commons Attribution License, which permits unrestricted use, distribution, and reproduction in any medium, provided the original work is properly cited.

This paper studies the energy efficiency (EE) maximization for an orthogonal frequency division multiple access (OFDMA) downlink network aided by a relay station (RS) with subcarrier pairing. A highly flexible transmission protocol is considered, where each transmission is executed in two time slots. Every subcarrier in each slot can either be used in direct mode or be paired with a subcarrier in another slot to operate in relay mode. The resource allocation (RA) in such a network is highly complicated, because it has to determine the operation mode of subcarriers, the assignment of subcarriers to users, and the power allocation of the base station and RS. We first propose a mathematical description of the RA strategy. Then, a RA algorithm is derived to find the globally optimum RA to maximize the EE. Finally, we present extensive numerical results to show the impact of minimum required rate of the network, the user number, and the relay position on the maximum EE of the network.

\section{Introduction}

With rapid growth of multimedia services, requirements for high-speed wireless communications are growing fast. To meet these requirements, telecom operators arrange a large number of base stations, which lead to a high amount of energy consumption [1]. To address this issue, many scholars have proposed energy-saving methods to minimize total energy consumption for a variety of wireless communication systems, such as Device-to-Device (D2D) communications, wireless sensor networks (WSNs), and cellular networks [2, 3]. Recently, energy efficiency- (EE-) based optimization design, which aims to maximize the EE defined as the number of transmitted bits per Joule total energy consumption, has attracted much interest from academia and industry [4-10].

Orthogonal frequency division multiple access (OFDMA) has been widely recognized as one of the dominant wireless technologies for high-data-rate wireless multimedia services. One of the main reasons behind this fact is that performance of OFDMA systems can be significantly improved by proper resource allocation (RA) when transmitter channel state information (CSI) is available [11-16]. Lately, relay-aided cooperation schemes have been widely used in combination with OFDMA networks to improve spectral efficiency. Under the constraint of guaranteeing users' communication rate, some works designed RA algorithms to minimize the total transmission power of networks [17-24]. In [17], to minimize the maximum value between transmission power of the BS and transmission power of all the RSs, Muller et al. designed a RA algorithm for decode-and-forward (DF) relay-aided OFDMA networks. In [21], knowing the relay selection, Huang et al. proposed an optimization algorithm of subcarriers and power allocation to minimize the total power of BS and all the RSs. Chen et al. designed a strategy of user assignment for subcarriers, RS's choice, and modulation scheme to minimize the total transmission power of networks for amplify-and-forward (AF) relay-aided downlink OFDMA networks [23]. The above works ignore the influence of circuit power of the BS and RSs, so these algorithms cannot ensure high $\mathrm{EE}$ of networks. 
It is interesting to further study how to improve EE of relay-aided OFDMA networks. When users lie outside the BS's radio coverage, EE maximized RA problems for OFDMA networks using subcarrier-pair-based DF protocols have been addressed in [25-29]. In these works, every subcarrier in the first time slot is paired with a subcarrier in the second time slot for the relay-aided transmission. In most cases, BS can also transmit messages to users directly; designing EE maximized RA algorithm for flexible transmission protocols is more meaningful. In [30-34], the authors adopted more flexible transmission protocols and proposed EE maximized RA algorithms for downlink OFDMA networks when the total transmission power is constrained to be smaller than a prescribed value. In this case, when the network reaches the maximum EE, the total communication rate might be too small to meet the needs of users.

In this paper, we focus on the optimum energy-efficient RA for downlink OFDMA with a RS using subcarrier-pairbased DF relaying, when the sum rate is constrained above a prescribed value. An opportunistic relay-aided transmission protocol is considered. User message bits are transmitted during two consecutive equal-duration time slots. In the first slot, the BS broadcasts OFDM symbols to RSs and users. In the second slot, subcarriers in direct mode can transmit to users directly; other subcarriers can be paired with subcarriers in first slot to transmit messages with the help of the RS. To be more specific, our contributions are summarized as follows:

(i) An EE maximized RA problem is formulated, and a polynomial-complexity algorithm is designed to find the optimum RA to maximize the EE based on the Dinkelbach method as well as the dual method to solve a subproblem.

(ii) Extensive numerical results are shown to exhibit the impact of system parameters (including minimum communication rate required by the network, RS deployment, and user number) on the network EE.

The rest of this paper is organized as follows. In the next section, the transmission protocol of the network is described. After that, the EE maximized RA algorithm is developed in Section 3. Numerical experiments are shown in Section 4. Finally, Section 5 concludes the paper.

Notations. $\mathscr{C}(x)=\log _{2}(1+x)$.

\section{Network Model and Transmission Protocol}

2.1. General Introduction of the Network and Protocol. We consider an OFDMA downlink network as shown in Figure 1. The network under consideration consists of a BS, a RS, and multiple users. Both the BS and the RS adopt OFDMA scheme using the same frequency band of bandwidth $B$ $\mathrm{Hz}$ and with $K$ subcarriers, which means that each OFDM symbol has a duration of $K / B$ seconds.

For illustration purpose, the channel coefficient and noise-power normalized channel gain at any subcarrier $k(k \in\{1, \ldots, K\})$ from BS to RS, from RS to any user $u$,

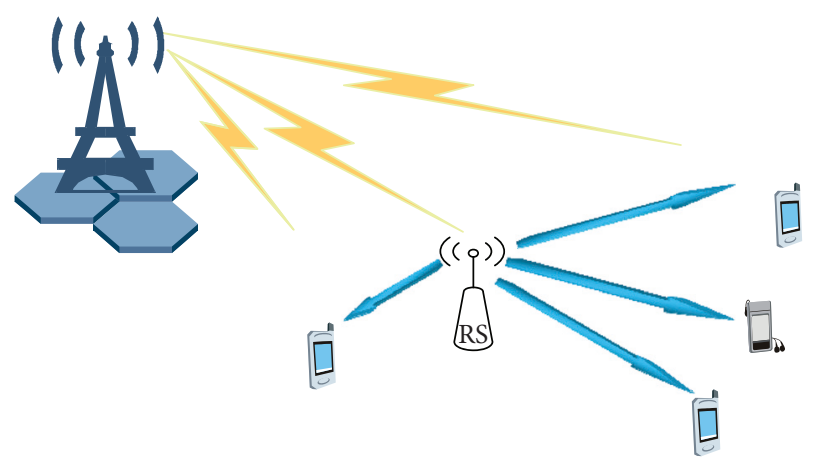

FIGURE 1: The relay-aided OFDMA downlink network under consideration.

TABLE 1: Channel coefficient and gain for subcarrier $k \in\{1, \ldots, K\}$.

\begin{tabular}{lcc}
\hline Channel for subcarrier $k$ & Coefficient & Gain \\
\hline BS to RS & $h_{s r}^{k}$ & $G_{s r}^{k}=\frac{\left|h_{s r}^{k}\right|^{2}}{\sigma^{2}}$ \\
BS to user $u$ & $h_{s u}^{k}$ & $G_{s u}^{k}=\frac{\left|h_{s u}^{k}\right|^{2}}{\sigma^{2}}$ \\
RS to user $u$ & $h_{r u}^{k}$ & $G_{r u}^{k}=\frac{\left|h_{r u}^{k}\right|^{2}}{\sigma^{2}}$ \\
\hline
\end{tabular}

and from BS to any user $u$ are defined in Table 1 , where $\sigma^{2}$ is assumed as the power of additive white Gaussian noise at each subcarrier.

The transmission protocol under consideration is carried out as follows. Each transmission needs two time slots denoted by slot-1 and slot-2, respectively. Each subcarrier in every slot can either operate in direct mode (it is used by the BS for transmission to a user directly) or be paired with a subcarrier in another slot to operate in relay mode (the relay helps the transmission as explained in Section 2.2). The protocol is illustrated by Figure 2. In the following subsections, the transmission procedures for the direct and relay mode are explained in detail.

2.2. Transmission for a Subcarrier Pair in Relay Mode. Suppose that a subcarrier $k$ in slot- 1 is paired with a subcarrier $l$ in slot-2 to operate in relay mode, and this relay-link is assigned to user $u$. Denote this subcarrier pair as $(k, l, u)$, which is shown in Figure 3. Over this link, the transmission procedure is carried out as follows.

In slot-1, the BS broadcasts a symbol $x$ to both the relay and the user with power $P_{1}$. The received signal at the relay is expressed as

$$
y_{r}=\sqrt{P_{1}} h_{s r}^{k} x+n_{r}
$$

and that at the user is expressed as

$$
y_{u, 1}=\sqrt{P_{1}} h_{s u}^{k} x+n_{u, 1}
$$

At the end of slot-1, the relay decodes $y_{r}$ and recovers $x$. To enable the relay to successfully decode the message bits, the 


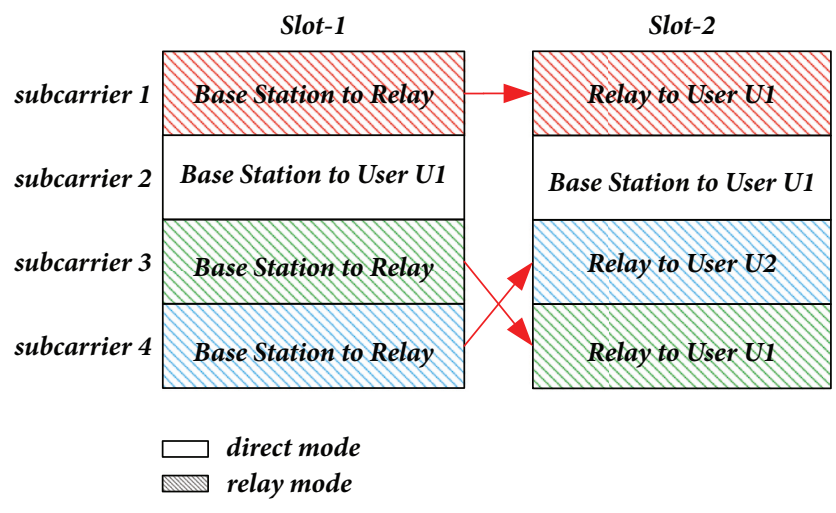

FIgURE 2: The transmission procedure over the subcarrier pair $(k, l, u)$.

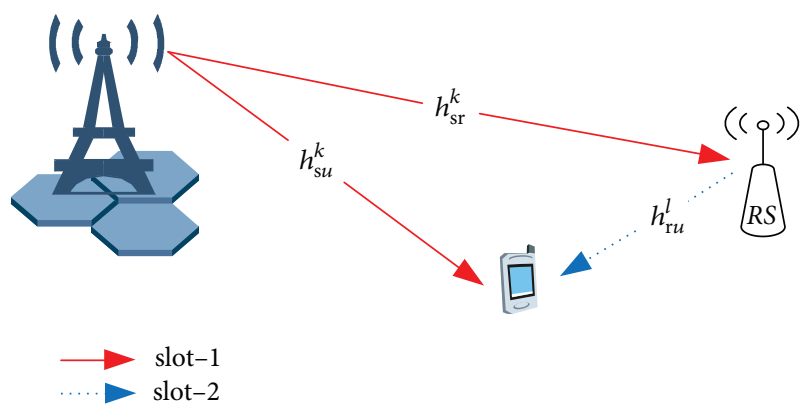

FIGURE 3: The transmission procedure over the subcarrier pair $(k, l, u)$.

maximum transmission rate is no greater than $\log _{2}\left(1+P_{1} G_{s r}^{k}\right)$ bits/symbol.

In slot-2, the relay simply emits $x$ at subcarrier $l$ to the user with power $P_{2}$. The received signal at the user is expressed as

$$
y_{u, 2}=\sqrt{P_{2}} h_{r u}^{l} x+n_{u, 2}
$$

At the end of slot-2, the source combines $y_{u, 1}$ and $y_{u, 2}$ with maximum-ratio combining (MRC) to maximize the received SNR. The final signal used for decoding at the user can be expressed as

$$
z=\alpha y_{u, 1}+\beta y_{u, 2}
$$

It can be shown that when $\alpha=\sqrt{P_{1}} h_{s u}^{k}$ and $\beta=\sqrt{P_{2}} h_{r u}^{l}{ }^{*}$, the MRC is achieved [35]. The maximum received SNR is

$$
\mathrm{SNR}_{k, l, u}=G_{s u}^{k} P_{1}+G_{r u}^{l} P_{2} \text {. }
$$

As a result, the maximum transmission rate over this relay link should be the minimum between the source-relay rate and the source-relay-user rate. It can be evaluated as

$$
C_{k l u}=\min \left\{\mathscr{C}\left(\mathrm{SNR}_{k l u}\right), \mathscr{C}\left(G_{s r}^{k} P_{1}\right)\right\}
$$

in the unit of bits/symbol.
Suppose that $P^{\prime}$ is the sum power of $P_{1}$ and $P_{2}$; the optimums $P_{1}$ and $P_{2}$ for maximizing the rate are the optimum solution for

$$
\begin{array}{ll}
\max _{P_{1}, P_{2}} & \min \left\{P_{1} G_{s r}^{k}, P_{1} G_{s u}^{k}+P_{2} G_{r u}^{l}\right\}, \\
\text { s.t. } & P_{1}+P_{2}=P^{\prime}, \quad P_{1} \geq 0, P_{2} \geq 0 .
\end{array}
$$

Using the same method as in [36], it can easily be shown that the optimums $P_{1}$ and $P_{2}$ are

$$
\begin{aligned}
& P_{1}= \begin{cases}\frac{G_{r u}^{l}}{\Delta_{u, k}+G_{r u}^{l}} P^{\prime} & \text { if } \min \left\{G_{s r}^{k}, G_{r u}^{l}\right\}>G_{s u}^{k}, \\
P^{\prime} & \text { if } \min \left\{G_{s r}^{k}, G_{r u}^{l}\right\} \leq G_{s u}^{k},\end{cases} \\
& P_{2}= \begin{cases}\frac{G_{s r}^{k}-G_{s u}^{k}}{\Delta_{u, k}+G_{r u}^{l}} P^{\prime} & \text { if } \min \left\{G_{s r}^{k}, G_{r u}^{l}\right\}>G_{s u}^{k}, \\
0 & \text { if } \min \left\{G_{s r}^{k}, G_{r u}^{l}\right\} \leq G_{s u}^{k},\end{cases}
\end{aligned}
$$

where $\Delta_{u, k}=G_{s r}^{k}-G_{s u}^{k}$.

The maximum rate associated with the above solution is equal to

$$
\begin{aligned}
& C_{k l u}= \frac{B}{2 K} \mathscr{C}\left(G_{k l u} P^{\prime}\right) \text { (bits/second), } \\
& G_{k l u}= \begin{cases}\frac{G_{s r}^{k} G_{r u}^{l}}{\Delta_{u, k}+G_{r u}^{l}} & \text { if } \min \left\{G_{s r}^{k}, G_{r u}^{l}\right\}>G_{s u}^{k}, \\
\min \left\{G_{s r}^{k}, G_{s u}^{k}\right\} & \text { if } \min \left\{G_{s r}^{k}, G_{r u}^{l}\right\} \leq G_{s u}^{k} .\end{cases}
\end{aligned}
$$

2.3. Transmission for a Subcarrier in Direct Mode. Every subcarrier in either slot- 1 or slot- 2 can be assigned to operate in direct mode. In this mode, a direct link from the BS to a certain user is formed at this subcarrier. Suppose that a subcarrier $k$ in either slot- 1 or slot- 2 is assigned to user $u$ and operates in the direct mode, and BS uses power $P$ for this subcarrier. Therefore, the average transmission rate over this direct link can be evaluated as

$$
\left.R(P)=\frac{B}{2 K} \mathscr{C}\left(G_{s u}^{k} P\right) \text { (bits/second }\right)
$$

\section{Energy-Efficient RA Algorithm Design}

Before data transmission, we assume that the BS controller knows all CSI, that is, $\left\{G_{s r}^{k}, G_{s u}^{k}, G_{r u}^{k} \mid \forall k, \forall u\right\}$. Based on the a priori knowledge, the BS runs an algorithm to find the optimum RA strategy to maximize the network EE.

To be more specific, the RA algorithm for the transmission protocol needs to be optimized:

(i) Subcarrier operation mode: how to decide whether each subcarrier should operate in direct or relay mode

(ii) Subcarrier pairing for relay mode: how to pair subcarriers in relay mode

(iii) Subcarrier assignment to users: how to allocate subcarriers to users 

(1) evaluate $G_{k l u}, \forall k, l, u$;
(2) $\theta_{\min }=0$; $\theta_{\max }$ is set by a large value; $\theta=\theta_{\max } ; \delta=10^{-3}$;
(3) while $|F(\theta)| \leq \delta$ do
(4) solve (P2) for $\mathbf{S}(\theta)$ using Algorithm 2;
(5) update $\theta$ by $\min \left\{\theta_{\text {max }}, \max \left\{R(\mathbf{S}(\theta)) / P(\mathbf{S}(\theta)), \theta_{\min }\right\}\right\}$;
(6) end while
(7) output $\theta$ as $\eta^{\star}$ and $\mathbf{S}(\theta)$ as the optimum RA strategy.

Algorithm 1: The algorithm to solve (P1).

(iv) Power allocation: how to allocate BS's and RS's transmission power for each subcarrier

To design the RA algorithm, we proceed as follows. First, a mathematical description of the RA strategy and network EE is proposed in Section 3.1 Then, a Dinkelbach-methodbased RA algorithm, namely, Algorithm 1, is designed in Section 3.2. Moreover, algorithms called by Algorithm 1 are designed in Sections 3.3 and 3.4.

3.1. Description of the RA Strategy and Network EE. We first define the following variables to describe the RA strategy:

(i) $t_{k l u} \in\{0,1\}, \forall k, l, u$ : indicating subcarrier $k$ in slot-1 is paired with subcarrier $l$ in slot- 2 when $t_{k l u}=1$.

(ii) $p_{k l u} \geq 0, \forall k, l, u$ : indicating the total transmission power (i.e., $P_{1}+P_{2}$ as mentioned in Section 2.2) for the subcarrier pair $(k, l, u)$.

(iii) $t_{k l a b} \in\{0,1\}, \forall k, l, a, b$ : indicating that subcarrier $k$ in slot- 1 and subcarrier $l$ in slot- 2 are, respectively, allocated to user $a$ and user $b$ when $t_{k l a b}=1$.

(iv) $p_{k l a b}^{1} \geq 0$ and $p_{k l a b}^{2} \geq 0$ : respectively indicating the BS's transmission power for subcarrier $k$ in slot- 1 and $l$ in slot-2 for the direct mode.

Let us define a RA strategy as $\mathbf{S}=\{\mathbf{I}, \mathbf{P}\}$, where $\mathbf{I}$ collects all indicator variables and $\mathbf{P}$ collects all power variables. A feasible $\mathbf{S}$ must satisfy

$$
\begin{aligned}
t_{k l u} & \in\{0,1\}, \\
t_{k l a b} & \in\{0,1\}, \\
\sum_{l}\left(\sum_{u} t_{k l u}+\sum_{a b} t_{k l a b}\right) & \leq 1, \quad \forall k, l, u, a, b, \\
\sum_{k}\left(\sum_{u} t_{k l u}+\sum_{a b} t_{k l a b}\right) & \leq 1, \quad \forall l, \\
p_{k l u} & \geq 0, \\
p_{k l a b}^{1} & \geq 0, \\
p_{k l a b}^{2} & \geq 0,
\end{aligned}
$$

$$
\forall k, l, u, a, b \text {, }
$$

where constraints (12) and (13) guarantee that each subcarrier in either slot- 1 or slot- 2 must operate in a single mode and be assigned to a single user.

For given $\mathbf{S}$, the network EE is formulated as

$$
\eta(\mathbf{S})=\frac{R(\mathbf{S})}{P(\mathbf{S})} \text { (bits/Joule), }
$$

where $R(\mathbf{S})$ represents the sum rate for the network:

$$
\begin{aligned}
& R(\mathbf{S})=\frac{B}{2 K}\left(\sum_{k l u} t_{k l u} \mathscr{C}\left(p_{k l u} G_{k l u}\right)\right. \\
& \left.\quad+\sum_{k l a b} t_{k l a b}\left(\mathscr{C}\left(p_{k l a b}^{1} G_{s u}^{k}\right)+\mathscr{C}\left(p_{k l a b}^{2} G_{s u}^{l}\right)\right)\right),
\end{aligned}
$$

and $P(\mathbf{S})$ is the sum power consumption for the network:

$$
\begin{aligned}
P(\mathbf{S})= & \frac{1+\alpha}{2}\left(\sum_{k l u} t_{k l u} p_{k l u}+\sum_{k l a b} t_{k l a b}\left(p_{k l a b}^{1}+p_{k l a b}^{2}\right)\right) \\
& +P_{\text {cir }},
\end{aligned}
$$

with $\alpha$ being the loss factor of the power amplifiers (PA) used by the BS and RS and $P_{\text {cir }}$ representing the total power consumption by the BS's and RS's circuit devices.

3.2. Dinkelbach-Method-Based RA Algorithm Design. We consider the EE maximization problem when the sum rate must be greater than a prescribed value $R_{\text {req }}$. This problem can be expressed as (P1):

$$
\begin{array}{ll}
\max _{\mathbf{S}} & \eta(\mathbf{S}) \\
\text { s.t. } & (11),(12),(13),(14) \\
& R(\mathbf{S}) \geq R_{\text {req }}
\end{array}
$$

It can be seen that solving problem (P1) is highly challenging due to the following reasons:

(i) $\eta(\mathbf{S})$ has a fractional structure, which is highly nonlinear.

(ii) (P1) is a mixed-integer problem containing both binary and continuous variables.

To solve (P1) for the optimum RA (denoted by $\mathbf{S}^{\star}$ ) and the maximum EE (denoted by $\eta^{\star}$ ), we make use of the Dinkelbach method. To be more specific, we define a parameter $\theta$ and a function $F(\theta)$ as the optimum objective value for the following problem (P2):

$$
\begin{array}{rl}
F(\theta)=\max _{\mathbf{S}} & R(\mathbf{S})-\theta P(\mathbf{S}) \\
\text { s.t. } & (11),(12),(13),(14),(18),
\end{array}
$$

whose optimum solution is $\mathbf{S}(\theta)$.

Based on Dinkelbach method, $\eta^{\star}$ must satisfy $F\left(\eta^{\star}\right)=0$, and $\mathbf{S}\left(\eta^{\star}\right)$ is the optimum solution for problem (P1). Moreover, $\eta^{\star}$ can be found by iterative procedures as elaborated in [6]. 


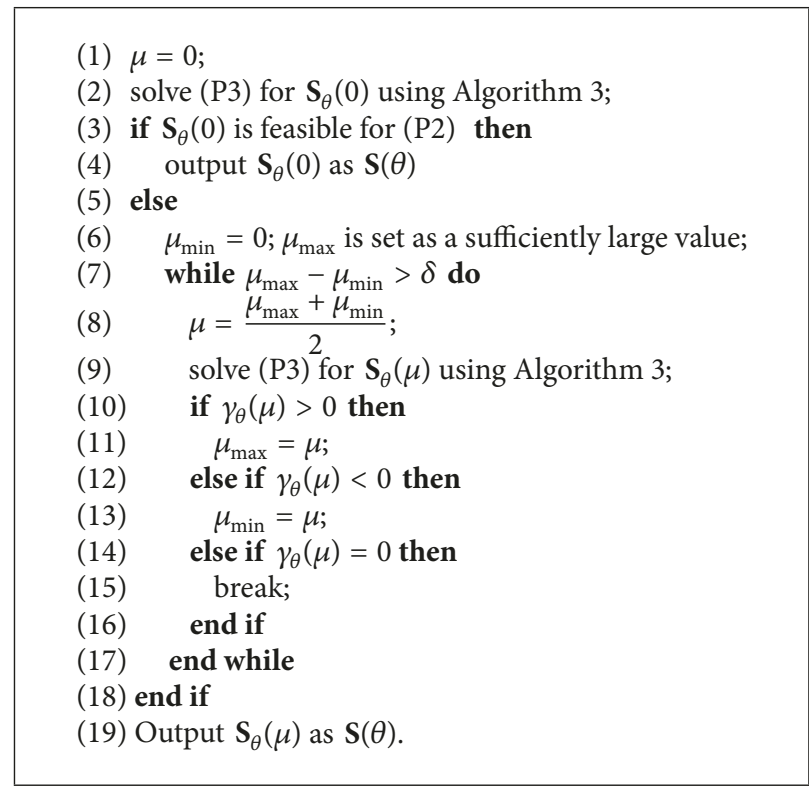

Algorithm 2: The algorithm to solve (P2).

(1) compute $p_{k l u}, p_{k l a b}^{1}, p_{k l a b}^{2} . \forall k, l, a, b, u$ with (25);

(2) compute $A_{k l u}, B_{k l a b}, \forall k, l, u, a, b$; and $C_{k l}, \forall k, l$;

(3) solve (P5) for its optimum solution $\left\{t_{k l}^{\star} \mid \forall k, l\right\}$ with the Hungarian algorithm;

(4) construct the optimum I for (P4) by assigning for every combination of $k$ and $l$, all entries in $\left\{t_{k l u}, t_{k l a b} \mid \forall k, l\right\}$ to zero, except for the one with the metric equal to $C_{k l}$ to $t_{k l}^{\star}$.

(5) $\mathbf{S}_{\mathbf{I}}=\left\{\mathbf{I}, \mathbf{P}_{\mathbf{I}}\right\}$ is output as $\mathbf{S}_{\theta}(\mu)$.

Algorithm 3: The algorithm to find $\mathbf{S}_{\theta}(\mu)$

Motivated by the above principle, the RA algorithm to solve (P1) for the EE maximized RA strategy is summarized in Algorithm 1. The iterative update of $\theta$ has a superlinear convergence rate [37]. We will elaborate on the design of Algorithm 2 to solve (P2) in Section 3.3.

3.3. Design of Algorithm 2 to Solve (P2). Using the same arguments as those in [38], we can show that (P2)'s duality gap is zero; therefore the dual method can be used to solve (P2). To this end, define

(i) $\mu$ as Lagrange multiplier for the constraint (18);

(ii) Lagrangian function

$$
L_{\theta}(\mu, \mathbf{S})=(R(\mathbf{S})-\theta P(\mathbf{S}))+\mu\left(R(\mathbf{S})-R_{\mathrm{req}}\right)
$$

(iii) Lagrange relaxation problem for (P2) as (P3):

$$
\begin{array}{ll}
\max _{\mathbf{S}} & L_{\theta}(\mu, \mathbf{S}) \\
\text { s.t. } & (11),(12),(13),(14)
\end{array}
$$

For (P3), we define its optimum solution as $\mathbf{S}_{\theta}(\mu)$ and its dual function as $d_{\theta}(\mu)=L_{\theta}\left(\mu, \mathbf{S}_{\theta}(\mu)\right)$. It can be shown that the dual function is convex of $\mu \geq 0$, and

$$
\gamma_{\theta}(\mu)=R\left(\mathbf{S}_{\theta}(\mu)\right)-R_{\text {req }}
$$

is a subgradient of $d_{\theta}(\mu)$ satisfying

$$
\forall \mu^{\prime}, d_{\theta}\left(\mu^{\prime}\right) \geq d_{\theta}(\mu)+\left(\mu^{\prime}-\mu\right) \gamma_{\theta}(\mu) \text {. }
$$

The key for the dual method is to find the dual optimum:

$$
\mu_{\theta}^{\star}=\arg \min _{\mu \geq 0} d_{\theta}(\mu),
$$

and then $\mathbf{S}_{\theta}\left(\mu_{\theta}^{\star}\right)$ is the optimum solution for (P2).

According to the dual method, $\mu_{\theta}^{\star}$ is the $\mu$ satisfying the following two conditions: (1) $\mu \gamma_{\theta}(\mu)=0$ (i.e., the complementary slackness condition) and (2) $\gamma_{\theta}(\mu) \geq 0$ (i.e., $\mathbf{S}_{\theta}(\mu)$ is feasible for (P2)). To find $\mu_{\theta}^{\star}$, we use the following method:

(i) First, we compute $\boldsymbol{S}_{\theta}(0)$. If $\gamma_{\theta}(0) \geq 0, \mu=0$ and $\mathbf{S}_{\theta}(0)$ satisfy the above conditions. Therefore, $\mathbf{S}_{\theta}(0)$ is the optimum for (P2). 
(ii) Otherwise, $\gamma_{\theta}(0)<0$ and $\mu_{\theta}^{\star}>0$ must hold. Note that $\gamma_{\theta}(\mu)$ is increasing of $\mu$. Once $\mu>0$ satisfying $\gamma_{\theta}(\mu)=0$ is found, $\mu$ and $\mathbf{S}_{\theta}(\mu)$ satisfy the above two conditions and hence can be taken as $\mu_{\theta}^{\star}$ and $\mathbf{S}(\theta)$, respectively. We will find $\mu>0$ satisfying $\gamma_{\theta}(\mu)=0$ with the bisection method.

To complete this subsection, the above procedures to solve (P2) are summarized in Algorithm 2 as follows. Algorithm 2 has a polynomial complexity with respect to $K$. We will elaborate on the design of Algorithm 3 to solve (P3) in Section 3.4.

3.4. Design of Algorithm 3 to Solve (P3). We show how to find $\mathbf{S}_{\theta}(\mu)$ as follows:

(i) First, the optimum $\mathbf{P}$ for (P3) with fixed I is found and denoted by $\mathbf{P}_{\mathbf{I}}$.

(ii) Second, define $\boldsymbol{S}_{\mathbf{I}}=\left\{\mathbf{I}, \mathbf{P}_{\mathbf{I}}\right\}$. Then we find the optimum I to maximize $L_{\theta}\left(\mu, \mathbf{S}_{\mathrm{I}}\right)$ subject to the constraints on I in (P3).

(iii) Finally, $\mathbf{S}_{\mathrm{I}}$ corresponding to this optimum I can be taken as $\mathbf{S}_{\theta}(\mu)$.

As for the first step, the elements in the optimum $\mathbf{P}_{\mathbf{I}}$ can be computed according to KKT conditions as follows [39]:

$$
\begin{array}{cl}
p_{k l u}=\Lambda\left(\theta, \mu, G_{k l u}\right), & \forall k, l, u \\
p_{k l a b}^{1}=\Lambda\left(\theta, \mu, G_{s u}^{k}\right), & \forall k, l, a, b \\
p_{k l a b}^{2}=\Lambda\left(\theta, \mu, G_{s u}^{l}\right), & \forall k, l, a, b,
\end{array}
$$

where

$$
\Lambda(\theta, \mu, G)=\left[\frac{(\mu+1) B \cdot \log _{2}(e)}{(1+\alpha) \theta \cdot K}-\frac{1}{G}\right]^{+}
$$

As for the second step, it can readily be shown that

$$
L_{\theta}\left(\mu, \mathrm{S}_{\mathrm{I}}\right)=-\mu R_{\mathrm{req}}+\sum_{k l u} t_{k l u} A_{k l u}+\sum_{k l a b} t_{k l a b} B_{k l a b}
$$

where

$$
\begin{aligned}
A_{k l u}= & \frac{(\mu+1) B}{2 K} \mathscr{C}\left(G_{k l u} \Lambda\left(\theta, \mu, G_{k l u}\right)\right) \\
& -\frac{\theta(1+\alpha)}{2} \Lambda\left(\theta, \mu, G_{k l u}\right) \\
B_{k l a b}= & \frac{(\mu+1) B}{2 K} \mathscr{C}\left(G_{s u}^{k} \Lambda\left(\theta, \mu, G_{s u}^{k}\right)\right) \\
& -\frac{\theta(1+\alpha)}{2} \Lambda\left(\theta, \mu, G_{s u}^{k}\right) \\
& +\frac{(\mu+1) B}{2 K} \mathscr{C}\left(G_{s u}^{l} \Lambda\left(\theta, \mu, G_{s u}^{l}\right)\right) \\
& -\frac{\theta(1+\alpha)}{2} \Lambda\left(\theta, \mu, G_{s u}^{l}\right)
\end{aligned}
$$

Finally, we find the optimum $\mathbf{I}$ for maximizing $L_{\theta}\left(\mu, \mathbf{S}_{\mathbf{I}}\right)$. This problem is equivalent to solving (P4):

$$
\begin{array}{rll}
\max _{\mathbf{I},\left\{t_{k l} l \forall k, l\right\}} & \sum_{k l} \sum_{u a b}\left(t_{k l u} A_{k l u}+t_{k l a b} B_{k l a b}\right) & \\
\text { s.t. } & t_{k l u} \geq 0, & \\
& t_{k l a b} \geq 0, & \\
& \\
& \sum_{l} t_{k l}=1, \quad \forall k, \\
& \sum_{k} t_{k l}=1, \quad \forall l, \\
t_{k l}=\sum_{u} t_{k l u}+\sum_{a b} t_{k l a b}, \quad \forall k, l, u, b,
\end{array}
$$

Note that

$$
\sum_{u a b}\left(t_{k l u} A_{k l u}+t_{k l a b} B_{k l a b}\right) \leq t_{k l} C_{k l}
$$

holds, where $C_{k l}=\max \left\{\max _{u} A_{k l u}, \max _{a, b} B_{k l a b}\right\}$. Call $A_{k l u}$ the metric for $t_{k l u}$ and $B_{k l a b}$ the metric for $t_{k l a b}$; the inequality is tightened when all entries of $\left\{t_{k l u}, t_{k l a b} \mid \forall u, a, b\right\}$ are assigned to zero, except that the one with the metric equal to $C_{k l}$ is assigned to $t_{k l}$.

Therefore, after problem (P5)

$$
\begin{aligned}
\max _{\left\{t_{k l} \mid \forall k, l\right\}} & \sum_{k l} t_{k l} C_{k l} \\
\text { s.t. } & (30),(31), \quad t_{k l} \in\{0,1\}, \forall k, l
\end{aligned}
$$

is solved for its optimum solution $\left\{t_{k l}^{\star} \mid \forall k, l\right\}$, an optimum (32) can be constructed by assigning for every combination of $k$ and $l$ all entries in $\left\{t_{k l u}, t_{k l a b} \mid \forall k, l\right\}$ to zero, except for the one with the metric equal to $C_{k l}$ to $t_{k l}^{\star}$.

Most interestingly, (P5) is a standard assignment problem; hence every entry in $\left\{t_{k l}^{\star} \mid \forall k, l\right\}$ is either 0 or 1 and $\left\{t_{k l}^{\star} \mid\right.$ $\forall k, l\}$ can be found efficiently by the Hungarian algorithm [40]. After knowing Hungarian algorithm, the optimum I can be constructed according to the way mentioned earlier.

Motivated by the above principle, the method to solve (P2) is summarized in Algorithm 3 as follows. The complexity of computing $A_{k l u}$ and $B_{k l a b}$ is $O\left(K^{2}\left(U+U^{2}\right)\right)$. We use the Hungarian algorithm to solve (P5); the complexity is $O\left(K^{3}\right)$. As a result, the complexity of Algorithm 3 is $O\left(K^{2}\left(U+U^{2}+\right.\right.$ $K)$ ).

\section{Numerical Experiments and Discussions}

We will first introduce system setup for numerical experiments, as well as two benchmark protocols for comparison purpose. Then, results and discussions are presented to show the impact of different parameters on the network EE. 
TABLE 2: Network Parameters.

\begin{tabular}{lcc}
\hline Meanings & Parameters & Values \\
\hline Bandwidth of the network & $B$ & $20 \mathrm{MHz}$ \\
Subcarriers' number & $K$ & 32 \\
Loss factor of the PA & $\alpha$ & 0.3 \\
Circuit power of BS and RS & $P_{\text {cir }}$ & $500 \mathrm{w}$ \\
Radius of UR & $R$ & $0.2 \mathrm{~km}$ \\
Distance between BS and the UR center & $D$ & $1.5 \mathrm{~km}$ \\
\hline
\end{tabular}

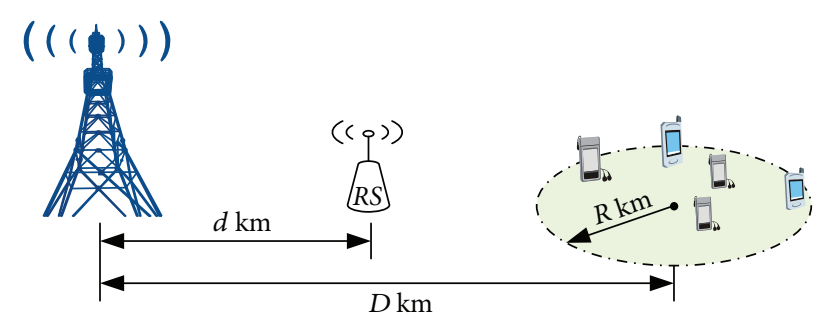

FIgURE 4: The downlink OFDMA system considered in numerical experiments.

4.1. System Setup and Benchmark Protocols. In numerical experiments, we consider the downlink OFDMA network with a RS exhibit in Figure 4. $U$ represents the number of users in service and they are randomly distributed in a circular region of radius $R \mathrm{~km}$. The $\mathrm{RS}$ is located between the $\mathrm{BS}$ and the user-region (UR) center, and the BS-RS distance is $d \mathrm{~km}$. The distance between the BS and the user-region center is $D \mathrm{~km}$. The bandwidth of the system is $B \mathrm{~Hz}$, and the OFDMA uses $K$ subcarriers. The network parameters are listed in Table 2.

The channels are independent of each other and are generated in the same way as in [7]. For every user $u$, the impulse response of the source-to- $u$ channel is modeled as a tapped delay line with $L=6$ taps, which are independently generated from circularly symmetric complex Gaussian distributions with zero mean and variance equal to $(1 / L)\left(d_{s u} / d_{\text {ref }}\right)^{-4}$, where $d_{\text {ref }}=1 \mathrm{~km}$ and $d_{s u}$ represents the source-to- $u$ distance. The source-to-relay and relay-to$u$ channels are generated in the same way, with each tap having variance as $(1 / L)\left(d_{s r} / d_{\text {ref }}\right)^{-4}$ and $(1 / L)\left(d_{r u} / d_{\text {ref }}\right)^{-4}$, respectively, where $d_{r u}$ represents the relay-to- $u$ distance. The CSI $\left\{h_{s r}^{k} \mid \forall k\right\},\left\{h_{s u}^{k} \mid \forall k, u\right\}$, and $\left\{h_{r u}^{k} \mid \forall k, u\right\}$ are computed by making $K$-point FFT over the impulse response of the associated channels.

In order to illustrate the benefit of optimized subcarrier pairing and opportunistic relaying, we also consider two other benchmark protocols, namely, BP-1 and BP-2. BP-1 is similar to the considered protocol, except that subcarrier $k$ in slot-1 can only be paired with subcarrier $k$ in slot- 2 in relay mode. BP-2 is a simplified version of the considered protocol, and the simplification lies in the fact that each subcarrier in every slot should be allocated to users in direct mode. The RA algorithms for both BP-1 and BP- 2 can be derived in the same way as that for the considered protocol, and therefore the derivation is omitted for the sake of clarity.
TABLE 3: Complexity comparison.

\begin{tabular}{lc}
\hline Algorithm & Complexity \\
\hline Proposed algorithm & $O\left(K^{2}\left(U+U^{2}+K\right)\right)$ \\
BP-1 & $O\left(K\left(U+U^{2}\right)\right)$ \\
BP-2 & $O(K U)$ \\
\hline
\end{tabular}

The complexity of the three algorithms is shown in Table 3. It can be seen that the proposed algorithm has the highest complexity, while the BP-2 algorithm has the lowest complexity.

4.2. Impact of $R_{\text {req }}$ on the Optimum EE and Corresponding Sum Rate. To show the influence of $R_{\text {req }}$ on the EE, we choose $U=$ 10 and $d=0.6 \mathrm{~km}$ and then evaluate the average optimum EE for every protocol over 1000 random channel realizations, when $R_{\text {req }}$ increases from 0 to $40 \mathrm{Mbits} / \mathrm{s}$. The results are shown in Figure 5.

Compared with BP-1 and BP-2, we can see that the proposed protocol and algorithm always correspond to a higher average EE as shown in Figure 5(a). Since BP-2 does not utilize opportunistic relaying as the proposed protocol and $\mathrm{BP}-1$, it is reasonable that BP-2 corresponds to much lower average EE. The proposed protocol can achieve higher EE than BP-1, because a subcarrier in every slot can be paired with the other slot's subcarrier freely.

Figure 5(a) also shows that the average EE of these methods decreases with the increase of $R_{\text {req. }}$. This is because the feasible set of the problem shrinks with the increase of $R_{\text {req }}$. From Figures 5(a), 5(b), and 5(c), when $R_{\text {req }}<20 \mathrm{Mbits} / \mathrm{s}$ and the average EE reaches the optimum value, the average communication rate is larger than $R_{\text {req }}$, the average total power remains stable, and the average EE of the network maintains high value. When $R_{\text {req }} \geq 20 \mathrm{Mbits} / \mathrm{s}$ and the average EE reaches the optimum value, the average communication rate is equal to $R_{\text {req }}$, the average total power increases rapidly, and the average EE of the network decreases. The above phenomenon indicates that the restricted condition $R(\mathbf{S}) \geq$ $R_{\text {req }}$ influences the choice of the optimum solution.

4.3. Impact of Relay Position on the Optimum EE and Corresponding Sum Rate. To show the impact of relay position on the EE, we choose $U=10$ and $R_{\text {req }}=20 \mathrm{Mbits} / \mathrm{s}$ and then evaluate the average optimum EE for every protocol over 1000 random channel realizations, when $d$ increases from 0.2 to $1.2 \mathrm{~km}$. The results are shown in Figure 6.

It is shown that the proposed protocol leads to a higher average EE than the BP-1 and BP-2 for every relay position. Moreover, the average EE improves as the RS moves towards the middle region between the BS and the users. This can be interpreted as follows. In theory, the optimum EE enhances if $\forall k, l, u, G_{k l u}$ is more likely to take a high value. Note that $G_{k l u}$ takes a high value only if both $G_{s r}^{k}$ and $G_{r u}^{l}$ are much higher than $G_{s u}^{k}$. When RS lies in the middle between the BS and the users' region, it is more likely to have $G_{s r}^{k}$ and $G_{r u}^{l}$, both much greater than $G_{s u}^{k}$, and thus $G_{k l u}$ is more likely to take a high value. Moreover, BP-2 is a direct transmission protocol; the 


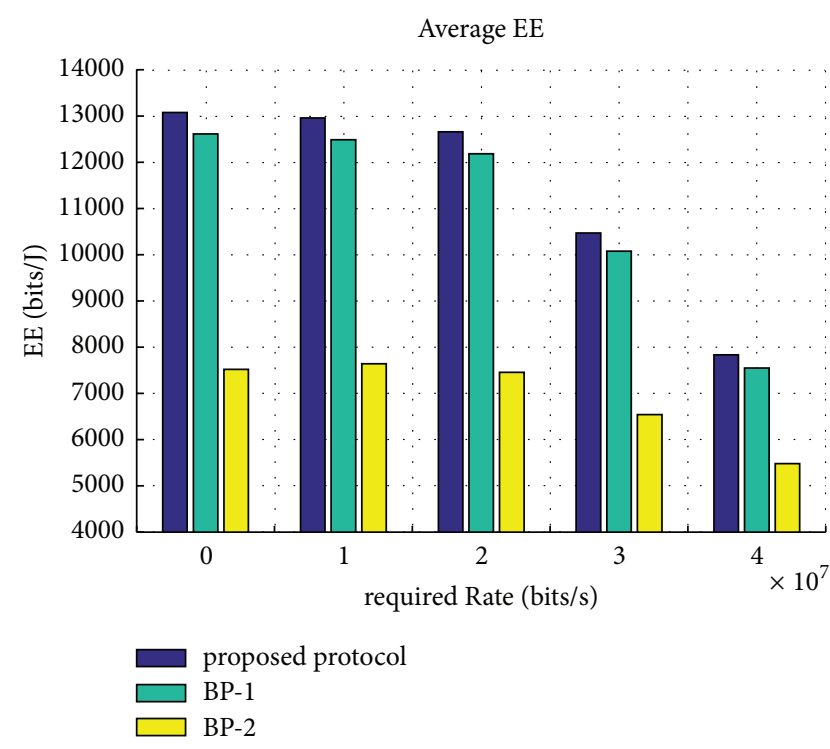

(a)

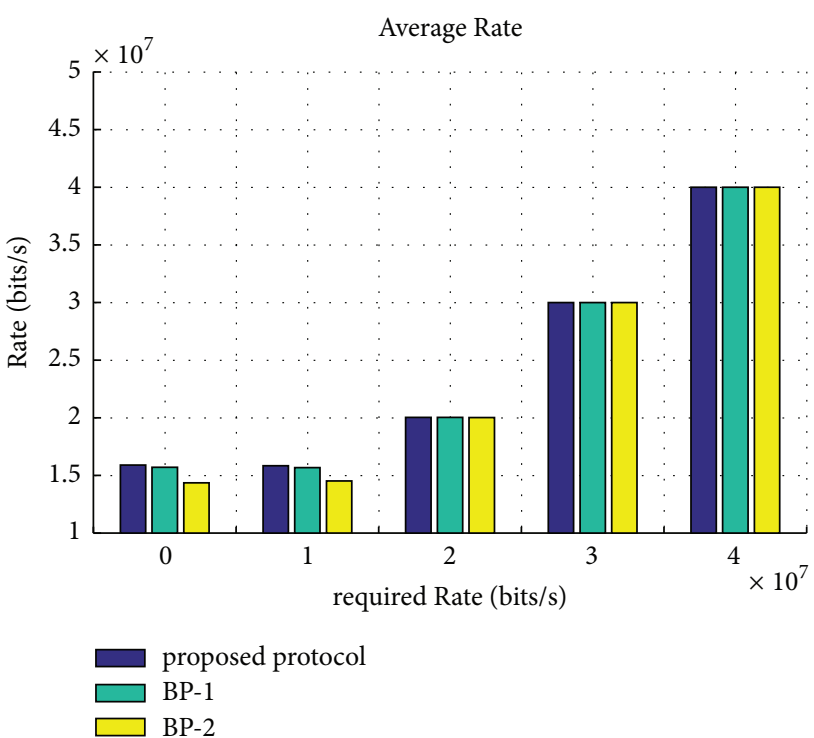

(b)

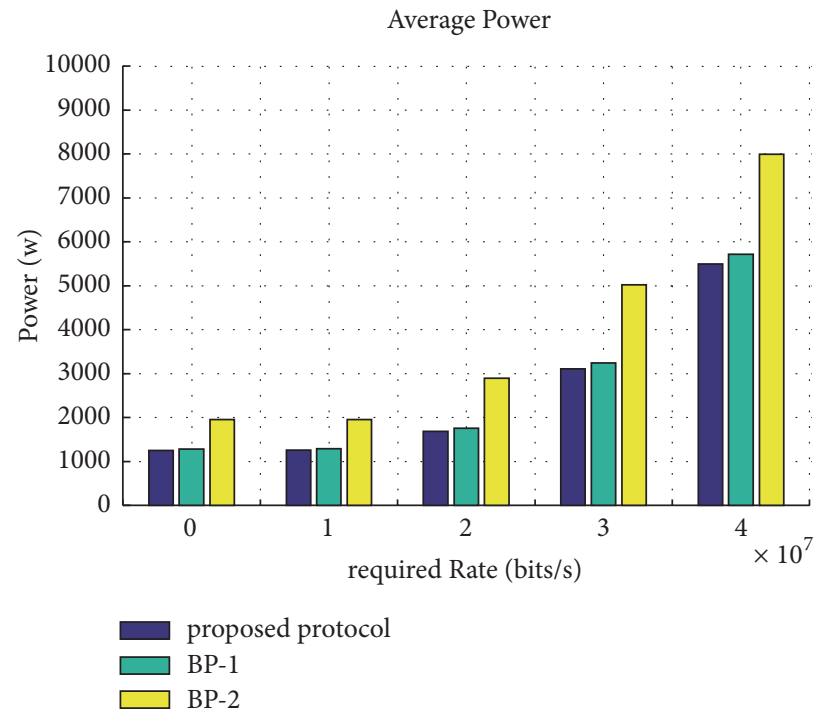

(c)

FIGURE 5: The average EE as the minimum rate changes.

RS does not help to transmit signals. It is reasonable that the average EE remains steady when the relay position changes.

4.4. Impact of User Number on the Optimum EE and Corresponding Sum Rate. To show the impact of user number on the EE, we choose $d=0.6 \mathrm{~km}$ and $R_{\text {req }}=20 \mathrm{Mbits} / \mathrm{s}$ and then evaluate the average optimum EE for every protocol over 1000 random channel realizations, when $U$ increases from 5 to 30 . The results are shown in Figure 7.

From Figure 7, we see that the average EEs of the three methods increase with the increase of user number. This is because when the number of users in the network increases, the subcarrier assignment has more flexibility. The numbers of $A_{k l u}$ and $B_{k l a b}$ increase with the increase of user number, which can improve the probability of $C_{k l}$ taking a larger value. In this way, the average $\mathrm{EE}$ of the network will be improved.

\section{Conclusions}

We have addressed an EE maximized RA problem for cooperative OFDM transmission using the improved DF protocol with optimized subcarrier pairing when the network's communication rate is larger than a required value. The subcarrier-pair-based opportunistic DF relay-aided protocol has two operation modes: direct mode and relay mode. This scheme improves the flexibility of the communication network. Subcarriers can choose the mode that can improve the network's EE to send messages. Based on the above protocol, 


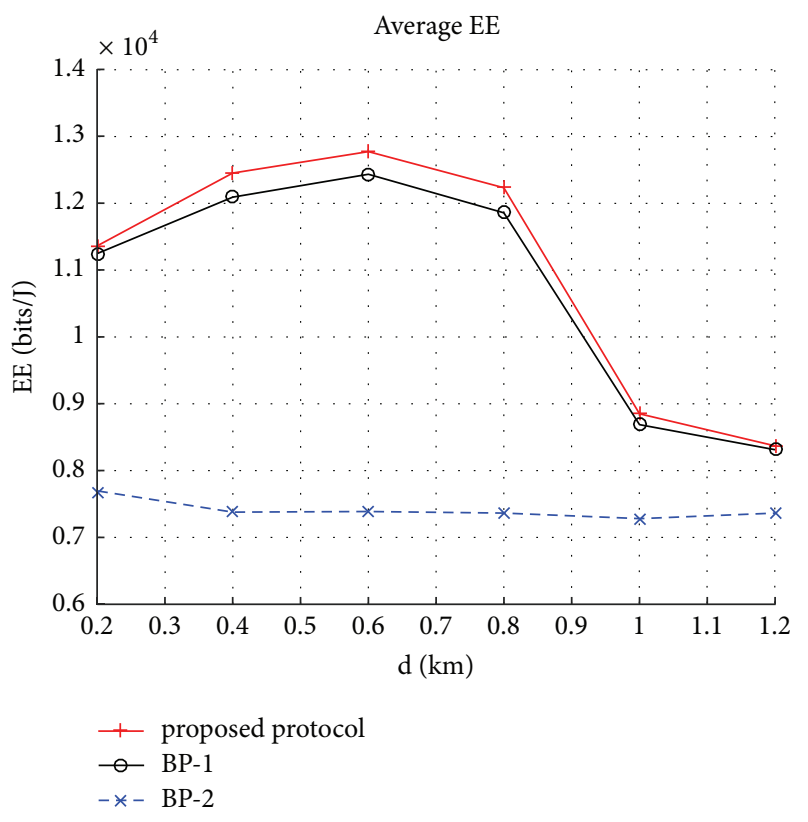

FIgure 6: The average EE as the relay position changes.

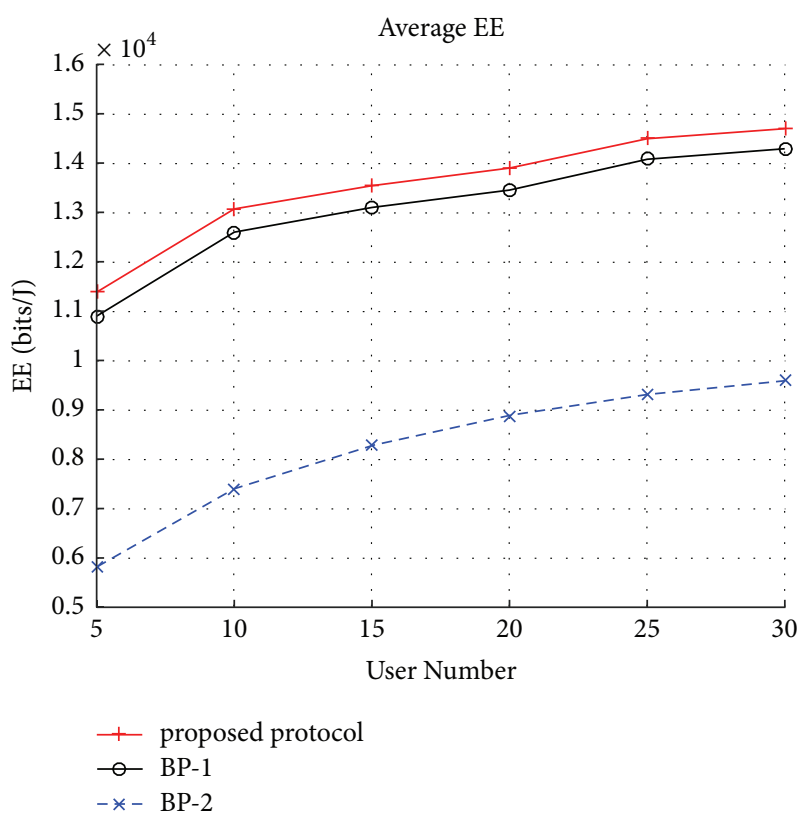

FIgURE 7: The average EE as the user number changes.

we formulate the optimization problem of maximizing the network's EE.

The problem is polynomial complexity, so we solve it with the following three steps. In the first step, we eliminate the fractional structure with the help of Dinkelbach method and transfer problem (P1) into problem (P2). In the second step, we get the Lagrangian function by using the dual method. In the third step, we use KKT conditions and Hungarian algorithm to solve the Lagrangian function. Then we can get the RA algorithm of maximizing the network's EE. Numerical experiments show that the proposed RA algorithm can improve the EE of the downlink OFDMA networks. And the experiments also illustrated the impact of minimum required communication rate, relay position, and the user number. Theoretical analysis has been presented to interpret what is observed in numerical experiments.

\section{Data Availability}

The data used to support the findings of this study are available from the corresponding author upon request.

\section{Conflicts of Interest}

The authors declare that they have no conflicts of interest.

\section{Acknowledgments}

This work was supported by the National Natural Science Foundation of China (Grants nos. 61671011, 61401266, 61501289, and 61701293), the National Science and Technology Major Project (Grant no. 2018ZX03001009), and research funds from Shanghai Institute for Advanced Communication and Data Science (SICS).

\section{References}

[1] G. Fettweis and E. Zimmermann, "ICT Energy Consumption Trends And Challenges," Journal Abbreviation, pp. 8-11, 2008.

[2] J. Wu, S. Rangan, and H. Zhang, Green Communications: Theoretical Fundamentals, Algorithms, and Applications, CRC Press, 2012.

[3] J. Wu, "Green wireless communications: From concept to reality [Industry perspectives]," IEEE Wireless Communications Magazine, vol. 19, no. 4, pp. 4-5, 2012.

[4] S. Cui, A. J. Goldsmith, and A. Bahai, "Energy-efficiency of MIMO and cooperative MIMO techniques in sensor networks," IEEE Journal on Selected Areas in Communications, vol. 22, no. 6, pp. 1089-1098, 2004.

[5] R. Bolla, R. Bruschi, F. Davoli, and F. Cucchietti, "Energy efficiency in the future internet: a survey of existing approaches and trends in energy-aware fixed network infrastructures," IEEE Communications Surveys \& Tutorials, vol. 13, no. 2, pp. 223-244, 2011.

[6] C. Isheden, Z. Chong, E. Jorswieck, and G. Fettweis, "Framework for link-level energy efficiency optimization with informed transmitter," IEEE Transactions on Wireless Communications, vol. 11, no. 8, pp. 2946-2957, 2012.

[7] T. Wang, "Energy-efficiency maximization for OFDMA networks with total power constraint," in Proceedings of the 2013 IEEE/CIC International Conference on Communications in China, ICCC 2013, pp. 357-361, China, August 2013.

[8] Z. Wang and L. Vandendorpe, "Subcarrier Allocation and Precoder Design for Energy Efficient MIMO-OFDMA Downlink Systems," IEEE Transactions on Communications, vol. 65, no. 1, pp. 136-146, 2017.

[9] S. Fu, H. Wen, J. Wu, and B. Wu, "Energy-Efficient Precoded Coordinated Multi-Point Transmission With Pricing Power Game Mechanism," IEEE Systems Journal, vol. 11, no. 2, pp. 578587, 2017.

[10] K. Yang, S. Martin, D. Quadri, J. Wu, and G. Feng, "EnergyEfficient Downlink Resource Allocation in Heterogeneous 
OFDMA Networks," IEEE Transactions on Vehicular Technology, vol. 66, no. 6, pp. 5086-5098, 2017.

[11] M. Mehrjoo, S. Moazeni, and X. Shen, "Resource allocation in OFDMA networks based on interior point methods," Wireless Communications and Mobile Computing, vol. 10, no. 11, pp. 14931508, 2010.

[12] C. Xiong, G. Y. Li, S. Zhang, Y. Chen, and S. Xu, "Energy- and spectral-efficiency tradeoff in downlink OFDMA networks," IEEE Transactions on Wireless Communications, vol. 10, no. 11, pp. 3874-3886, 2011.

[13] T. Wang and L. Vandendorpe, "On the SCALE algorithm for multiuser multicarrier power spectrum management," IEEE Transactions on Signal Processing, vol. 60, no. 9, pp. 4992-4998, 2012.

[14] Q. Wu, W. Chen, M. Tao, J. Li, H. Tang, and J. Wu, "Resource allocation for joint transmitter and receiver energy efficiency maximization in downlink OFDMA systems," IEEE Transactions on Communications, vol. 63, no. 2, pp. 416-430, 2015.

[15] J. Tang, D. K. C. So, E. Alsusa, K. A. Hamdi, and A. Shojaeifard, "On the energy efficiency-spectral efficiency tradeoff in MIMOOFDMA broadcast channels," IEEE Transactions on Vehicular Technology, vol. 65, no. 7, pp. 5185-5199, 2016.

[16] G. Zhang, J. Hu, W. Heng, and G. Wang, "Distributed power and resource allocation for weighted sum energy-efficiency maximization in OFDMA smallcell network," in Proceedings of the 27th IEEE Annual International Symposium on Personal, Indoor, and Mobile Radio Communications, PIMRC 2016, pp. 15, September 2016.

[17] C. Muller, A. Klein, and F. Wegner, "Dynamic subcarrier, bit and power allocation in OFDMA-based relay networks," in 12th International OFDM Workshop, pp. 1-5, 2007.

[18] T. Girici, "Joint power, subcarrier and subframe allocation in Multihop relay networks," International Journal of Communication Systems, vol. 22, no. 7, pp. 835-855, 2009.

[19] T. Wang and L. Vandendorpe, "WSR maximized resource allocation in multiple DF relays aided OFDMA downlink transmission," IEEE Transactions on Signal Processing, vol. 59, no. 8, pp. 3964-3976, 2011.

[20] F. Lu, C. Liu, and H.-A. Zhao, "On the joint optimal power allocation for $\mathrm{df}$ relaying and beamforming communication systems," IEICE Transactions on Communications, vol. E95-B, no. 3, pp. 889-897, 2012.

[21] B. Huang, X. Fang, and Y. Chen, "Joint energy saving resource allocation and user scheduling in OFDMA relay networks," in Proceedings of the IEEE/CIC International Conference on Communications in China, pp. 484-490, IEEE, Xian, China, August 2013.

[22] B. Huang, X. Fang, Y. Zhao, Y. Chen, and R. He, "Dynamic energy saving subcarrier, bit and power allocation in OFDMA relay networks," China Communications, vol. 10, no. 4, pp. 7987, 2013.

[23] Y. Chen, X. Fang, and B. Huang, "Energy-efficient relay selection and resource allocation in nonregenerative relay OFDMA systems," IEEE Transactions on Vehicular Technology, vol. 63, no. 8, pp. 3689-3699, 2014.

[24] X. Chen, F. He, L. Xiao, and S. Zhou, "Joint subcarrier and power allocation for DF-based multiuser two-way relay networks," China Communications, vol. 14, no. 6, Article ID 7961373, pp. 179-188, 2017.

[25] Y. Li, W. Wang, J. Kong, and M. Peng, "Subcarrier pairing for amplify-and-forward and decode-and-forward OFDM relay links," IEEE Communications Letters, vol. 13, no. 4, pp. 209-211, 2009.

[26] T. Wang, "Weighted sum power minimisation for multichannel decode-and-forward relaying," IEEE Electronics Letters, vol. 48, no. 7, pp. 410-411, 2012.

[27] E. S. Hassan, "Energy-efficient hybrid opportunistic cooperative protocol for single-carrier frequency division multiple access-based networks," IET Communications, vol. 6, no. 16, pp. 2602-2612, 2012.

[28] G. Zhou, T. Wang, Y. Wu, G. Zheng, and G. Yang, "EnergyEfficient Power Allocation for Decode-and-Forward OFDM Relay Links," in Mobile and Wireless Technologies 2016, vol. 391 of Lecture Notes in Electrical Engineering, pp. 13-24, Springer Singapore, Singapore, 2016.

[29] W. Yang and X. Zhao, "Resource allocation in two-way OFDMbased cognitive radio networks with QoE and power consumption guarantees," EURASIP Journal on Wireless Communications and Networking, vol. 2017, no. 1, article no. 216, 2017.

[30] W. Yang, L.-H. Li, W.-L. Sun, and Y. Wang, "Energy-efficient relay selection and optimal relay location in cooperative cellular networks with asymmetric traffic," Journal of China Universities of Posts and Telecommunications, vol. 17, no. 6, pp. 80-88, 2010.

[31] C. Y. Ho and C.-Y. Huang, "Energy efficient subcarrierpower allocation and relay selection scheme for OFDMA-based cooperative relay networks," in Proceedings of the 2011 IEEE International Conference on Communications, pp. 1-6, Japan, June 2011.

[32] K. T. K. Cheung, S. Yang, and L. Hanzo, "Achieving maximum energy-efficiency in multi-relay OFDMA cellular networks: a fractional programming approach," IEEE Transactions on Communications, vol. 61, no. 7, pp. 2746-2757, 2013.

[33] Z. Song, Q. Ni, K. Navaie, S. Hou, S. Wu, and X. Sun, "On the spectral-energy efficiency and rate fairness tradeoff in relayaided cooperative OFDMA systems," IEEE Transactions on Wireless Communications, vol. 15, no. 9, pp. 6342-6355, 2016.

[34] F. Heliot and R. Tafazolli, "Optimal Energy-Efficient Joint Resource Allocation for Multi-Hop MIMO-AF Systems," IEEE Transactions on Communications, vol. 64, no. 9, pp. 3655-3668, 2016.

[35] D. Tse and P. Viswanath, Fundamentals of Wireless Communication, Cambridge University Press, Cambridge, UK, 2005.

[36] T. Wang, F. Glineur, J. Louveaux, and L. Vandendorpe, "Weighted sum rate maximization for downlink OFDMA with subcarrier-pair based opportunistic DF relaying," IEEE Transactions on Signal Processing, vol. 61, no. 10, pp. 2512-2524, 2013.

[37] W. Dinkelbach, "On nonlinear fractional programming," Management Science, vol. 13, no. 7, pp. 492-498, 1967.

[38] Y. Wu and T. Wang, "Energy-efficient resource allocation for OFDM transmission with opportunistic DF relaying," in Proceedings of the 2014 IEEE/CIC International Conference on Communications in China, ICCC 2014, pp. 570-575, chn, October 2014.

[39] S. Boyd and L. Vandenberghe, Convex Optimization, Cambridge University Press, 2013.

[40] H. W. Kuhn, "The Hungarian method for the assignment problem," Naval Research Logistics Quarterly, vol. 2, pp. 83-97, 1955. 


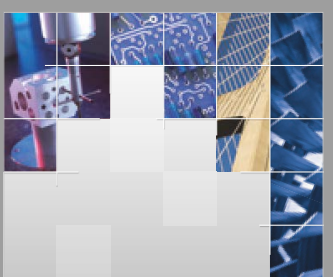

\section{Enfincering}
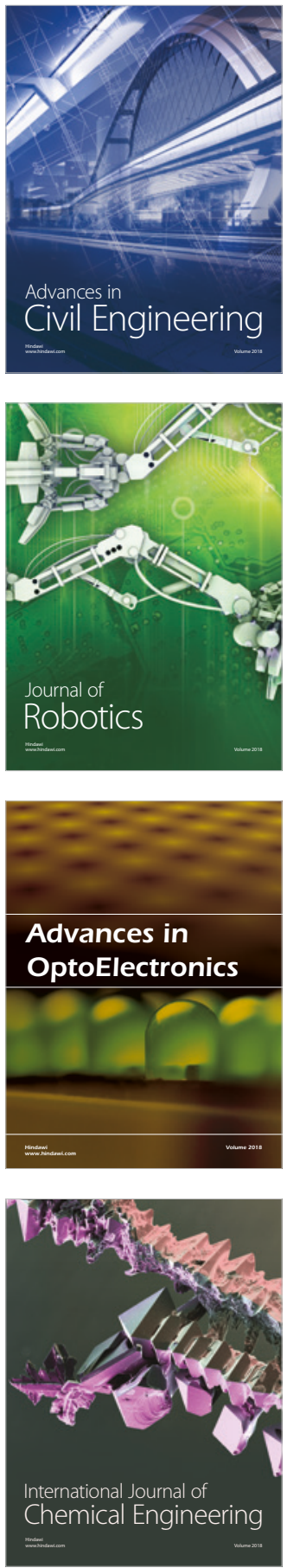

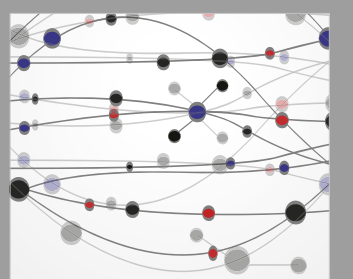

\section{Rotating \\ Machinery}

The Scientific World Journal

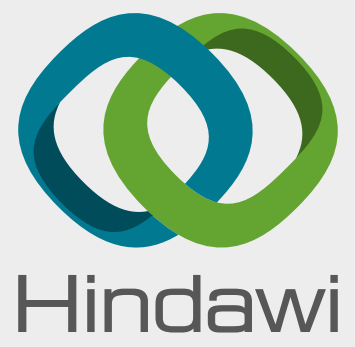

Submit your manuscripts at

www.hindawi.com
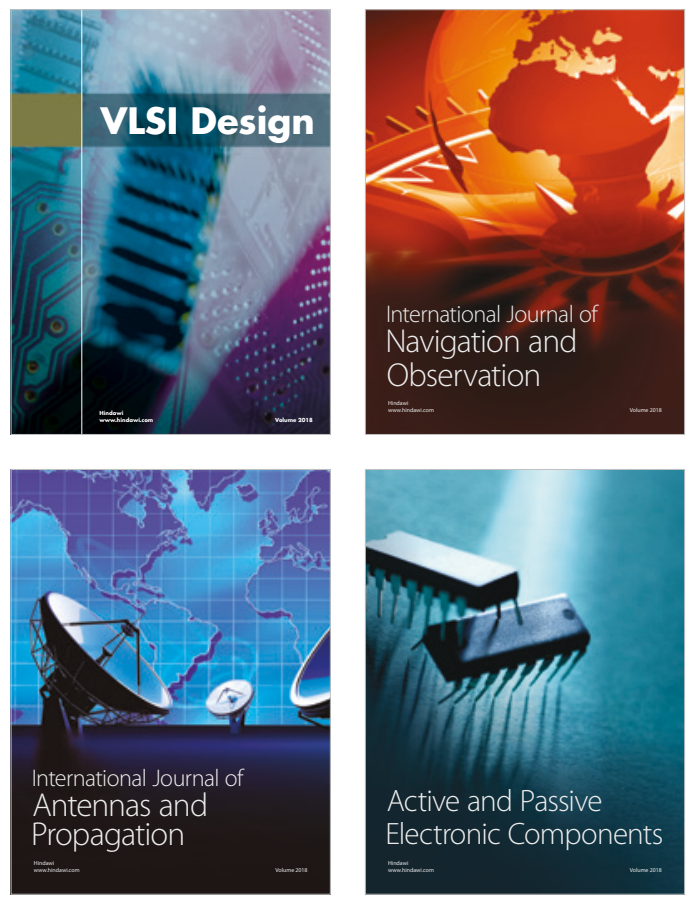
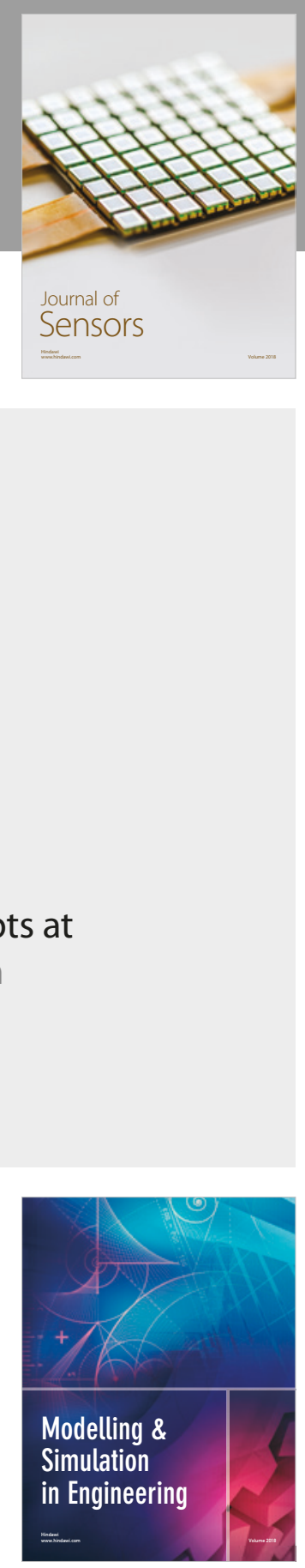

\section{Advances \\ Multimedia}
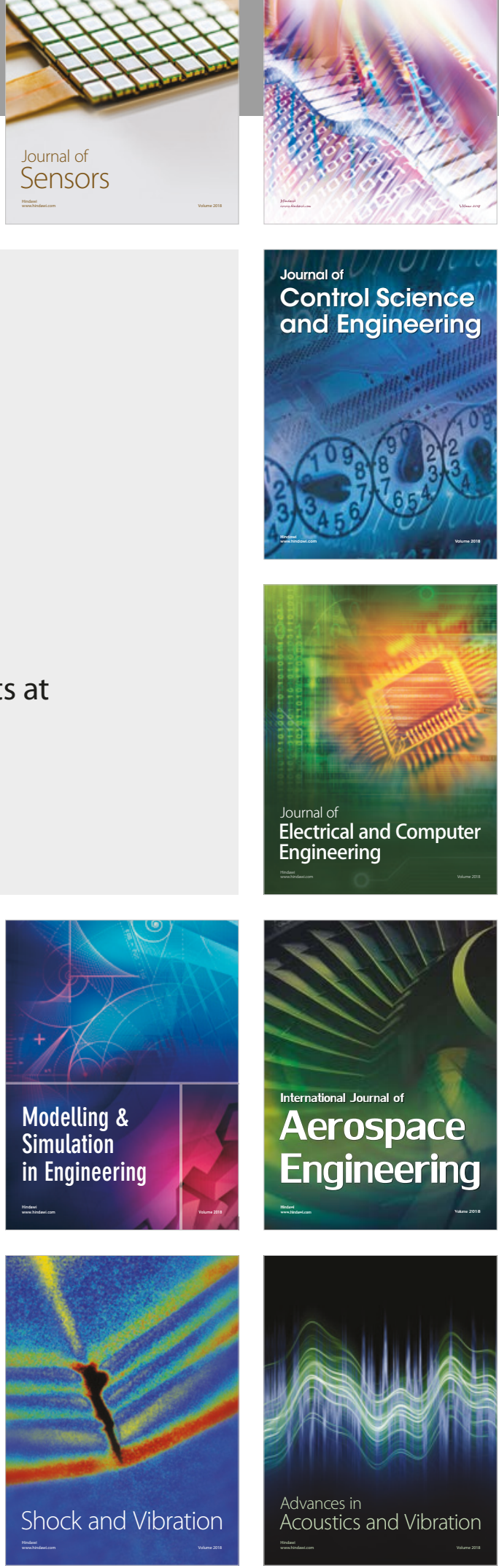